\title{
An Unexpected Finding: Neuroendocrine Neoplasm in the Descending Duodenum
}

\section{Ting-Ting Zhou ${ }^{1,2} \mid$ Li-Hong Du ${ }^{1,2}$ | Wei Liu ${ }^{1,2 *}$}

*Correspondence: Wei Liu, Ph.D., Institute of Digestive Disease, China Three Gorges University, 8 Daxue Road, Yichang 443000, China

Address: ${ }^{1}$ Institute of Digestive Disease, China Three Gorges University, Yichang, China; ${ }^{2}$ Department of Gastroenterology, Yichang Central People's Hospital, Yichang, China

e-mail $\bowtie$ : liuwei@ctgu.edu.cn

Received: 19 February 2021; Accepted: 22 February 2021

\section{Clinical Image}
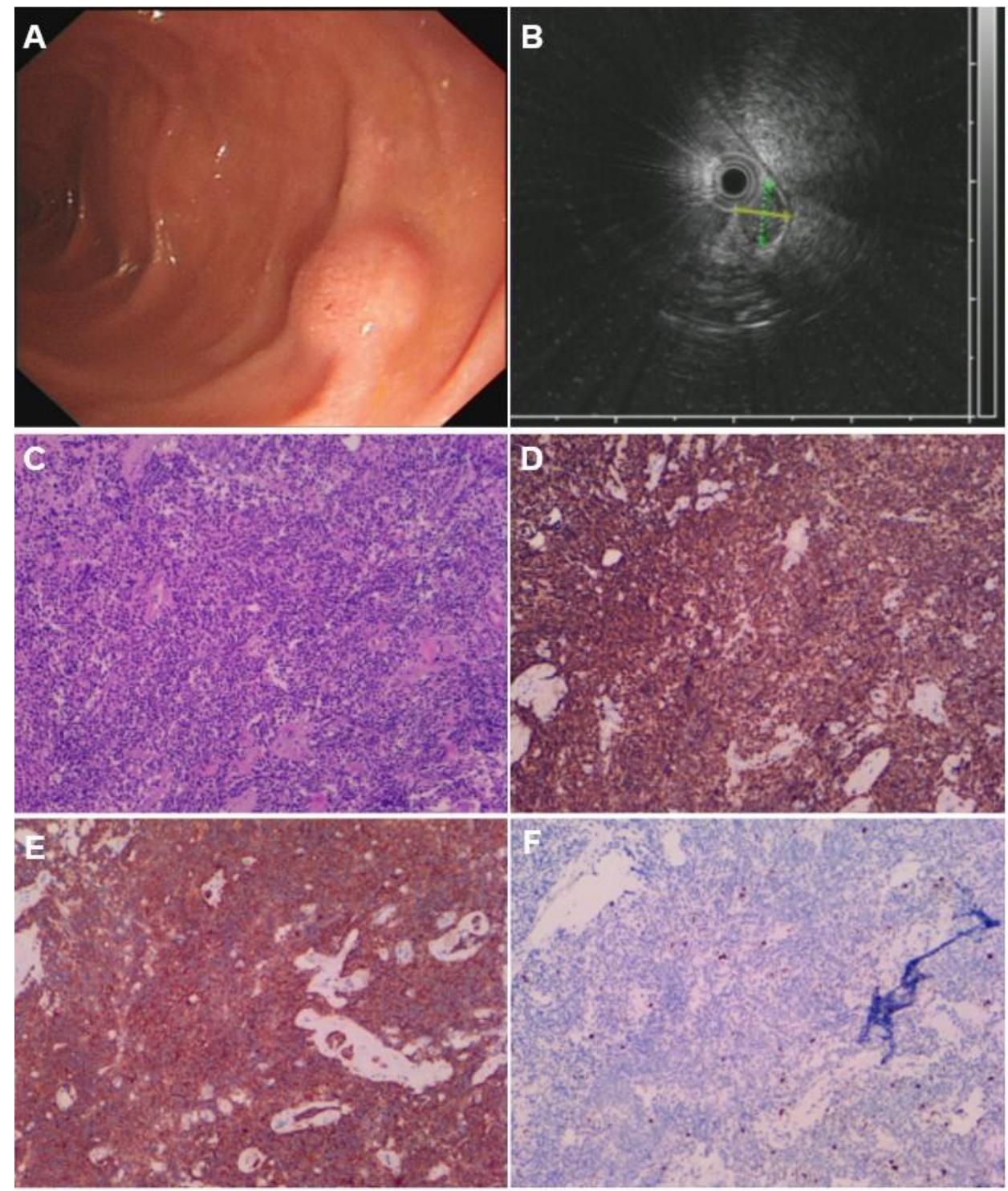

Figures A-F 
A 51-year-old female was admitted to the gastroenterology clinic in October 2020 because of a 2year history of epigastric pain unrelated to food intake. No diarrhea was noted. Her documented medical history was notable for well-controlled hypertension. Her physical examination and Helicobacter pylori antigen detection showed no remarkable abnormalities. A computed tomography scan of the abdomen revealed a $0.5 \times 0.5 \mathrm{~cm}$ well-defined hyperattenuating structure in the wall of the descending duodenum, which was confirmed by esophagogastroduodenoscopy demonstrating a $0.5 \mathrm{~cm}$ nodule in the descending duodenum (Fig. A). The lesion was located within the deep mucosa and submucosa without invasion of adjacent structures detected by endoscopic ultrasound (Fig. B). Endoscopic submucosal dissection was performed to remove the nodule despite the high risk for postoperative complications. Microscopic examination of the specimen by hematoxylin and eosin staining (Fig. C) and the lesion staining strongly immunoreactive for synaptophysin (Fig. D) and chromogranin (Fig. E) by immunohistochemistry were consistent with a well-differentiated grade 2 neuroendocrine neoplasm, which was further defined by a low proliferation index Ki67 around 3\% in neoplastic cells (Fig. F). No etiology for patient's epigastric pain was discovered on esophagogastroduodenoscopic examination. Duodenal neuroendocrine tumors consist of $2 \%$ of all gastrointestinal neuroendocrine tumors and $1 \%$ of all duodenal tumors, which are very rare (Baliss et al., 2021). The rarity of duodenal neuroendocrine tumors coupled with the absence of neuroendocrine clinical syndromes creates a noteworthy diagnostic challenge (Delle et al., 2016). Patients with nonfunctional and nonmetastatic duodenal neuroendocrine tumors should be considered for resection regardless of tumor size (Sato et al., 2016).

\section{References}

Baliss M, Bilal M, Merwat S. Nothing but NET: A Case of Neuroendocrine Tumor in the Duodenal Bulb. Clin Gastroenterol Hepatol 2021;19: e6.

Delle Fave G, Sundin A, Taal B, Ferolla P, Ramage JK, Ferone D, Ito T, Weber W, Zheng-Pei Z, De Herder WW, Pascher A. ENETS Consensus Guidelines Update for Gastroduodenal Neuroendocrine Neoplasms. Neuroendocrinology 2016; 103: 119-124.

Sato Y, Hashimoto S, Mizuno KI, Takeuchi M, Terai S. Management of gastric and duodenal neuroendocrine tumors. World J Gastroenterol 2016; 22: 6817-6828. 